\title{
Caution in the age of optimism; a salient lesson in advanced melanoma
}

\author{
Hayley N. Roberts ${ }^{1}$, Andrew Haydon ${ }^{1,2}$ \\ ${ }^{1}$ Department of Medical Oncology, Alfred Hospital, Melbourne, Australia; ${ }^{2}$ Monash University, Melbourne, Australia \\ Correspondence to: Associate Professor Andrew Haydon. Department of Medical Oncology, Alfred Hospital, Melbourne, Australia. \\ Email: Andrew.haydon@monash.edu. \\ Provenance and Peer Review: This article was commissioned by the editorial office, Annals of Translational Medicine. The article did not undergo \\ external peer review. \\ Comment on: Long GV, Dummer R, Hamid O, et al. Epacadostat plus pembrolizumab versus placebo plus pembrolizumab in patients with \\ unresectable or metastatic melanoma (ECHO-301/KEYNOTE-252): a phase 3, randomised, double-blind study. Lancet Oncol 2019;20:1083-97.
}

Submitted Dec 31, 2019. Accepted for publication Jan 15, 2020.

doi: 10.21037/atm.2020.01.125

View this article at: http://dx.doi.org/10.21037/atm.2020.01.125

Tumours can express genes that suppress host immune responses to their own tumour antigens, and the modulation of these immune responses has become a target for cancer therapeutics. Indoleamine 2,3-dioxygenase (IDO) is an enzyme that catalyses the conversion of tryptophan to kynurenine. This is the first step in the kynurenine metabolism pathway, which contributes to local immunosuppression in the tumour microenvironment by inhibition of effector $\mathrm{T}$ cells and activation of regulatory $\mathrm{T}$ cells (1). IDO expression in melanoma has been associated with worsened prognosis (2), and IDO1 inhibitors such as epacadostat have become the subject of recent trials in patients with stage III and IV melanoma, with the hope that they may be combined with other current treatments to improve outcomes.

The PD-1 (programmed death protein 1) inhibitors pembrolizumab and nivolumab have become standard therapies in advanced melanoma, after they were shown to improve overall survival compared to the CTLA-4 (cytotoxic T-lymphocyte-associated protein 4 inhibitor) ipilimumab $(3,4)$. Given that tumours may use multiple pathways to evade immune surveillance, there has been interest in combination immunotherapy with CTLA-4 and PD-1 inhibitors. While there was slightly numerically superior overall survival in patients treated with nivolumab plus ipilimumab compared to nivolumab alone in CheckMate 067, the trial was not designed or powered to compare these two treatments (4). On the other hand, patients with asymptomatic brain metastases appear to get considerable benefit from combination immunotherapy, with intracranial response rates around $50 \%$ to ipilimumab plus nivolumab compared to $20 \%$ with single-agent nivolumab $(5,6)$. A deterrent to combination immunotherapy is the significantly higher rate of treatment-related adverse events; grade 3 or 4 events occur in $59 \%$ of patients treated with nivolumab plus ipilimumab, compared to $21 \%$ of patients on nivolumab monotherapy, and $28 \%$ of patients on ipilimumab monotherapy (4).

Given the toxicity and lack of clear benefit of combining PD-1 inhibitors with CTLA-4 inhibitors, interest has turned to examining novel agents in combination with an anti PD-1 backbone. Many recently completed, ongoing and planned trials are looking to find therapeutic combinations that are superior to single agent anti PD-1 without adding greatly to the toxicity. Examples of agents being tested include oncolytic viruses (such as T-Vec), other checkpoint inhibitors (LAG-3, TIM-3), co-stimulatory agents (GITR, OX40) and many other strategies. One of these strategies has been to combine IDO1 inhibitors with either pembrolizumab or nivolumab. Initial trials suggested that the combination of IDO1 inhibitors and PD-1 inhibitors might confer a survival benefit. A singlearm phase I/II trial of 64 patients with advanced melanoma combining pembrolizumab with the oral IDO1 inhibitor epacadostat showed encouraging results, with overall response rates of $56 \%$, and median progression-free survival of 12.4 months (7). As a historical comparator, response rates were $36 \%$ and median progression-free survival was 
4.1 months in patients taking 3-weekly pembrolizumab in the KEYNOTE-006 trial (8). A phase I/II trial evaluating the combination of nivolumab with epacadostat in 50 patients found an overall response rate of $62 \%$. Forty-eight percent of these patients had grade 3 or 4 adverse events at a dose of $300 \mathrm{mg}$ bd, compared to $13 \%$ for the $100 \mathrm{mg}$ bd dose (9).

Disappointingly, the first large phase III trial combining a PD-1 inhibitor with epacadostat has shown no benefit over PD-1 inhibitor monotherapy. ECHO-301/KEYNOTE-252 was an international, placebo-controlled trial which randomly assigned 706 patients with unresectable stage III or IV melanoma to receive either epacadostat $100 \mathrm{mg}$ orally twice daily plus pembrolizumab $(n=354)$ or placebo plus pembrolizumab $(n=352)$, having first been stratified by PD-L1 expression and BRAF ${ }^{V 600}$ mutation status. Prespecified primary endpoints were progression-free survival and overall survival in the intention-to-treat population. With regard to power, the study had approximately $98 \%$ power to detect a progression free-survival hazard ratio of 0.65 favouring epacadostat over placebo, and $79 \%$ power to detect a favourable overall survival hazard ratio of 0.7 . Notably, the study was only powered for one-sided analysis, suggesting a high degree of optimism that the addition of epacadostat would confer a significant survival advantage.

Long et al. found that patients treated with epacadostat plus pembrolizumab had no significant difference in progression-free survival or overall survival compared to those treated with placebo plus pembrolizumab. Median progression-free survival was 4.7 months (95\% CI, 2.9-6.8) for epacadostat plus pembrolizumab, and 4.9 months (95\% CI, 2.9-6.8) for placebo plus pembrolizumab. The study was stopped after the second interim analysis due to the primary objectives of prolonged progression-free or overall survival not being met in the epacadostat group. Due to the early cessation and resultant short follow-up of 12.4 months, median overall survival has not been reached in either treatment group, with the hazard ratio for overall survival of 1.13 (95\% CI, 0.86-1.49; one-sided $\mathrm{P}=0.81$ ).

The lack of a survival benefit for patients taking epacadostat in addition to pembrolizumab was surprising, given initial promising results in the phase I/II study. However, the phase III trial population may have differed from this group in a number of important ways. Uncontrolled selection of patients with more favourable characteristics may have occurred in the phase I/II trials. The exclusion criteria differed slightly, but potentially significantly; the phase I/II trials excluded patients previously treated with BRAF-targeted treatment, who were included in the phase III trial. Historically, treatment naïve patients have had a superior response to immunotherapy (3). The phase I/II trial was small, non-randomised and easily weighted towards favourable patient selection, and on the basis of promising results from this trial a much larger, more resource-intensive phase III study was undertaken.

Further research into IDO1 inhibitors might examine higher doses, combination blockade with IDO2 and TDO (tryptophan 2,3-dioxygenase) inhibitors, selection of patients on the basis of IDO biomarkers, combination with other immunotherapy agents, or use in other tumour types. There is uncertainty as to whether the $100 \mathrm{mg}$ bd dose was sufficient; a dose of $300 \mathrm{mg}$ bd have previously been suggested as the dose that would inhibit $>90 \%$ of IDO1 activity (10). Additionally, measurements of IDO1 activity in response to different doses have so far been based on the proxy serum markers of plasma kynurenine levels and kynurenine/tryptophan ratios, rather than on intratumoral IDO1. Potentially useful analyses of pharmacokinetics and pharmacodynamics were not performed on the trial population due to a decision from the sponsor against devoting more resources to this negative study. There is a concern that perhaps IDO1 inhibition is easily bypassed by rapidly mutating tumours, that can instead express other tryptophan-degrading enzymes such as IDO2 and TDO (tryptophan 2,3-dioxygenase) (11). These enzymes could be targets for co-inhibition in future trials.

A lesson learnt from this noteworthy phase III negative trial is the need for larger, randomised phase II trials prior to proceeding to costly phase III studies, understanding that small benefits may well be missed in phase II studies. Significant patient expectations and hope, economic resources and highly skilled researchers' and clinicians' time is invested into an international multicentre trial of 706 patients. The opportunity cost of running such a trial is weighed against investing those resources into other worthy research and clinical care.

With regard to implications for the current standard of care, this study reinforces that an appropriate treatment for most patients with metastatic melanoma remains single-agent PD-1 inhibitor. The implications for future research are wide-reaching. The results from the ECHO301/KEYNOTE-252 study led to the announcement from Incyte (epacadostat's drugmaker) to cease phase III clinical development of combination epacadostat with antiPD-1/PD-L1 immunotherapy. This meant converting two pembrolizumab combination trials in lung cancer to phase II status, closing enrolment into four trials 
with pembrolizumab and two trials with nivolumab, and cancelling a planned trial with durvalumab (12). There are also implications for how trials are conducted and progressed. Significant confidence exists in the field of melanoma research, attributable to large positive trials that have significantly prolonged many patients' lives. However, caution in progression to large phase III trials is still warranted in this age of optimism, given the implications of haste for patients, researchers and clinicians.

\section{Acknowledgments}

Funding: None.

\section{Footnote}

Conflicts of Interest: Both authors have completed the ICMJE uniform disclosure form (available at http://dx.doi. org/10.21037/atm.2020.01.125). Associate Professor AH is a member of advisory boards for Amgen, BMS, Merck and Pierre-Fabre. HNR has no conflicts of interest to declare.

Ethical Statement: The authors are accountable for all aspects of the work in ensuring that questions related to the accuracy or integrity of any part of the work are appropriately investigated and resolved.

Open Access Statement: This is an Open Access article distributed in accordance with the Creative Commons Attribution-NonCommercial-NoDerivs 4.0 International License (CC BY-NC-ND 4.0), which permits the noncommercial replication and distribution of the article with the strict proviso that no changes or edits are made and the original work is properly cited (including links to both the formal publication through the relevant DOI and the license). See: https://creativecommons.org/licenses/by-nc-nd/4.0/.

\section{References}

1. Munn DH, Mellor AL. Indoleamine 2,3-dioxygenase and tumor-induced tolerance. J Clin Invest 2007;117:1147-54.

2. Brody JR, Costantino CL, Berger AC, et al. Expression of indoleamine 2,3-dioxygenase in metastatic malignant melanoma recruits regulatory $\mathrm{T}$ cells to avoid immune detection and affects survival. Cell Cycle 2009;8:1930-4.

3. Robert C, Ribas A, Schachter J, et al. Pembrolizumab versus ipilimumab in advanced melanoma (KEYNOTE-006): post-hoc 5 -year results from an openlabel, multicentre, randomised, controlled, phase 3 study. Lancet Oncol 2019;20:1239-51.

4. Wolchok JD, Chiarion-Sileni V, Gonzalez R, et al. Overall Survival with Combined Nivolumab and Ipilimumab in Advanced Melanoma. N Engl J Med 2017;377:1345-56.

5. Tawbi HA, Forsyth PA, Algazi A, et al. Combined Nivolumab and Ipilimumab in Melanoma Metastatic to the Brain. N Engl J Med 2018;379:722-30.

6. Long GV, Atkinson V, Lo S, et al. Combination nivolumab and ipilimumab or nivolumab alone in melanoma brain metastases: a multicentre randomised phase 2 study. Lancet Oncol 2018;19:672-81.

7. Hamid O, Gajewski TF, Frankel AE, et al. 1214OEpacadostat plus pembrolizumab in patients with advanced melanoma: Phase 1 and 2 efficacy and safety results from ECHO-202/KEYNOTE-037. Ann Oncol 2017;28.

8. Schachter J, Ribas A, Long GV, et al. Pembrolizumab versus ipilimumab for advanced melanoma: final overall survival results of a multicentre, randomised, open-label phase 3 study (KEYNOTE-006). Lancet 2017;390:1853-62.

9. Daud A, Saleh MN, Hu J, et al. Epacadostat plus nivolumab for advanced melanoma: Updated phase 2 results of the ECHO-204 study. J Clin Oncol 2018;36:9511.

10. Beatty GL, O'Dwyer PJ, Clark J, et al. First-in-Human Phase I Study of the Oral Inhibitor of Indoleamine 2,3-Dioxygenase-1 Epacadostat (INCB024360) in Patients with Advanced Solid Malignancies. Clin Cancer Res 2017;23:3269-76.

11. Muller AJ, Manfredi MG, Zakharia Y, et al. Inhibiting IDO pathways to treat cancer: lessons from the ECHO301 trial and beyond. Semin Immunopathol 2019;41:41-8.

12. Incyte Corporation. Incyte Reports 2018 First-Quarter Financial Results and Updates on Key Clinical Programs. Available online: https://investor.incyte.com/newsreleases/news-release-details/incyte-reports-2018-firstquarter-financial-results-and-updates

Cite this article as: Roberts HN, Haydon A. Caution in the age of optimism; a salient lesson in advanced melanoma. Ann Transl Med 2020;8(11):720. doi: 10.21037/atm.2020.01.125 